\title{
Prognostic value and clinicopathological features of PD-1/PD-L1 expression with mismatch repair status and desmoplastic stroma in Chinese patients with pancreatic cancer
}

\author{
Yu Wang ${ }^{1,2,3,4, *}$, Jiacheng Linn ${ }^{3,4, *}$, Jiujie Cui $2,3,4, *$, Ting Han ${ }^{1,2,3,4}$, Feng Jiao ${ }^{2,3,4}$, Zhuo \\ Meng $^{1,2,3,4}$, Liwei Wang ${ }^{1,2,3,4}$ \\ ${ }^{1}$ Department of Medical Oncology and Pancreatic Cancer Center, Renji Hospital, School of Medicine, Shanghai Jiao Tong \\ University, Shanghai 200000, China \\ ${ }^{2}$ Department of Medical Oncology and Pancreatic Cancer Center, Shanghai General Hospital, School of Medicine, Shanghai \\ Jiao Tong University, Shanghai 201620, China \\ ${ }^{3}$ Shanghai Key Laboratory of Pancreatic Disease, Shanghai 201620, China \\ ${ }^{4}$ State Key Laboratory of Oncogene and Related Genes, Shanghai Cancer Institute, Renji Hospital, Shanghai Jiao Tong \\ University School of Medicine, Shanghai 200032, China \\ *These authors are contributed equally to this work
}

Correspondence to: Liwei Wang, email: liweiwang@shsmu.edu.cn

Keywords: $P D-1 / P D-L 1$, mismatch repair enzymes, pancreatic cancer, prognosis

Received: September 27, $2016 \quad$ Accepted: December 16, 2016

Published: December 21, 2016

\section{ABSTRACT}

Pancreatic cancer (PC) is a highly lethal cancer. Thus, the immune molecular markers which help to select PC patients are especially important. In this study, we aimed at systematically analyzing the expression of MLH1, MSH2, PD-L1 and PD-1, investigate their clinical significance and prognostic value. We found that high expression of PD-L1 on cancer cell membranes correlated with lymph node metastasis $(P=0.033)$ and strongly correlated with poor-differentiation $(P=0.008)$; high expression of PD-1 on cell membranes of T-cells correlated with well-differentiation $(P=0.018)$ and strongly correlated with advanced $T$ stage $(P=0.004)$; high $P D-1$ expression was associated with a significantly superior OS and was an independent prognostic factor $(P=\mathbf{0 . 0 3 1})$. Then we found an inverse correlation between MSH2 expression and PD-L1 expression (Spearman correlation coefficient $r=-0.295$, $P=0.004)$. In subgroup analyses, we observed that PD-1 expression level was associated with OS only at low PD-L1 expression subgroup $(P=0.021)$. Finally, when we stratified the cases into four subgroups based on PD-1 expression and stroma density, we found that patients with high PD-1 expression and dense stroma had a better OS, while patients with low PD-1 expression and moderate stroma showed a worst outcome. Our result may provide more effective molecular markers for immunotherapeutic strategies of PC patients in clinical practice.

\section{INTRODUCTION}

Pancreatic cancer (PC) is a highly lethal cancer, with a 5-year survival of approximately $5 \%[1-2]$. The potentially resectable of PC is less than $20 \%$, for the diagnosis of pancreatic cancer is often difficult, and most patients are diagnosed at an advanced stage. However, even in rescetable cases, the median survival time is only $15-18$ months. Recently, surgery, radiotherapy and chemotherapy for pancreatic cancer have been improved, but the effect was limited. Thus, it is very important to find more effective molecular markers for the treatment and prognosis of $\mathrm{PC}$ patients in clinical practice.

Program death ligand 1 (PD-L1) is a member of the B7 family of immune-regulatory cell-surface proteins. As a receptor of PD-L1, programmed death-1 (PD-1) is typically expressed on activated T cells. PD-1/PD-L1 axis plays an important role in the negative regulation of cell-mediated immune responses as an immune checkpoint. The expression of PD-1/PD-L1 is up-regulated in many tumors and their microenvironment [3-5]. PC is characterised by an abundant desmoplastic stroma which 
contains dense activated stellate cells. PC is considered as a non-immunogenic tumor with few effector $\mathrm{T}$ cells infiltrating into the tumor tissue. The stroma of PC is poor-vascularized and often prevents $\mathrm{T}$-cell from infiltrating [6-9]. Although the antibodies of PD-1/ PD-L1 have shown improving outcome in patients with melanoma, renal cell carcinoma, non-small lung cancer, the results of treating pancreatic cancer with single-agent immune checkpoint inhibitors have been disappointing [10-13]. However, anti-tumor activity was preliminarily reported for some PC patients at the 2014 ASCO annual meeting. Thus, the immune molecular markers which help to select PC patients are especially important [14].

High PD-L1 expression was firstly suggested to predict the response to anti-PD-1/PD-L1 antibody therapies. Then, pembrolizumab has been reported to be highly effective in gastrointestinal cancers in patients with mismatch repair deficiencies [15-16]. Deficiencies of mismatch repair enzymes (MLH1, MSH2, MSH6, and PMS2) were originally identified by Dr. Henry Lynch [17]. Meanwhile, mismatch repair deficiency (MMR-D) cancer appears to be more antigenic than mismatch repair proficient (MMR-P) cancer and has a special susceptibility to immunotherapy. Since somatic mutations have the potential to create immunogenic neoantigens, patients with mismatch repair defects, which promote somatic mutations, may have increased intratumoral effector $\mathrm{T}$ cell responses to the neoantigens [15-16]. Thus, effective immune-based therapeutic strategies are highly demanded for the majority of malignancies that are naturally immune quiescent.

Although many studies showed that the expression of PD-1 and PD-L1 was correlated with the clinical outcome in several malignancies including PC [18-19], some studies did not find any prognostic impact of PD-1/PD-L1[20]. Also, the factors which affected the expression of PD-1/ PD-L1 in PC were not fully illustrated. Moreover, the role of mismatch repair enzymes in this disease and correlation with PD-1/PD-L1 expression remains largely unknown. In this study, we firstly aim to evaluate the correlation of clinical/pathological characteristics and the expression of PD-1/PD-L1, MLH1, MSH2 respectively and demonstrated the prognostic role of them in a tissue microarray (TMA) including 94 well-documented, clinically annotated PC specimens. Then, we evaluated the correlation of PD-1, PD-L1, MLH1, MSH2 and desmoplastic stroma density and investigated their clinical significance in PC progression.

\section{RESULTS}

\section{Expression of PD-1, PD-L1, MLH1 and MSH2 in PC tissues and staining characteristics}

Immunohistochemistry (IHC) and haematoxylin-eosin (HE) staining results are summarized in Table 1 and Figure 1. At high magnification, MLH1 and MSH2 were distributed in the nucleus, while PD-1/PD-L1 was located on cell membranes. MLH1 was highly expressed in 63.8\% (60/94) of PC samples (Figure 1A, 1B), while MSH2 was highly expressed in $75.5 \%$ (71/94) of PC samples (Figure 1C, 1D). High expression of PD-1 was observed in 46.8\% (44/94) on tumor infiltrating lymphocytes (TILs) (Figure 1E, 1F). In contrast, PD-L1 was expressed on cell membranes of cancer cells with high expression in $28.7 \%$ (27/94) of PC tissues (Figure $1 \mathrm{G}, 1 \mathrm{H}$ ). The density of tumor stroma was evaluated by haematoxylin-eosin staining. Dense stroma was observed in 55.3\% (52/94) of cases (Figure 1I, 1J, Supplementary Figure S1).

\section{Correlations between MLH1/MSH2/PD-1/PD-L1 and clinicopathological characteristics}

The relationships of the expression levels of MLH1, MSH2, PD-1 and PD-L1 with clinicopathological features of PC were evaluated. For MLH1 and MSH2, as summarized in Table 2, we did not observe any correlation between their expression levels and clinicopathological characteristics (Table 2). High expression of PD-L1 on cancer cell membranes correlated with lymph node metastasis $(P=0.033)$ and strongly correlated with poor-differentiation $(P=0.008)$, but did not correlate with patient's gender, age, tumor location, T stage, distant metastasis, vascular invasion and nervous invasion $(P>0.05)$ (Table 3$)$. Meanwhile, high expression of PD-1 on cell membranes of TILs correlated with well-differentiation $(P=0.018)$ and strongly correlated with advanced T stage $(P=0.004)$, but did not correlate with patient's gender, age, tumor location, $\mathrm{N}$ stage, distant metastasis, vascular invasion and nervous invasion $(P>0.05)$ (Table 3$)$. The relationships of the stroma density with clinicopathological features of PC were evaluated by haematoxylin-eosin staining. As summarized in Table 3, no correlation was observed between stroma density and patients' clinicopathologic features.

\section{Prognostic impact of MLH1, MSH2, PD-1, PD- L1 and stroma density in PC}

Kaplan-Meier analysis and log-rank test were used to evaluate the prognostic impact of MLH1, MSH2, PD-1, PD-L1, stroma density and clinicopathologic characteristics on patient survival. The log-rank test results showed that a high PD-1+ TILs expression had a significantly superior overall survival (OS) (log-rank test: $P=0.004)$ (Figure $2 \mathrm{E}$ ), but we failed to observe any prognostic impact of stroma density and the expression of MLH1, MSH2 and PD-L1 (Figure 2A-2D). Then we used the Cox regression model to evaluate the correlation between prognostic factors and OS. As shown in Table 4, high PD-1+ TILs expression, well differentiation and no vascular invasion were associated with a significantly superior OS $(P=0.008, \mathrm{HR}=0.441,95 \%$ CI $0.242-0.804)$, while poor differentiation and vascular invasion were associated with a poor OS $(P=0.026, \mathrm{HR}=1.963$, 
Table 1: Results of MLH1, MSH2, PD-L1 and PD-1 IHC and HE staining

\begin{tabular}{lccccc}
\hline Maker & $\begin{array}{c}\text { MLH1 } \\
\boldsymbol{n}(\mathbf{\%})\end{array}$ & $\begin{array}{c}\text { MSH2 } \\
\boldsymbol{n}(\mathbf{\%})\end{array}$ & $\begin{array}{c}\text { PD-L1 } \\
\boldsymbol{n}(\mathbf{\%})\end{array}$ & $\begin{array}{c}\text { PD-1 } \\
\boldsymbol{n}(\mathbf{\%})\end{array}$ & $\begin{array}{c}\text { Stroma }^{\text {a }} \\
\boldsymbol{n}(\mathbf{\%})\end{array}$ \\
\hline Low score & $34(36.2 \%)$ & $23(24.5 \%)$ & $67(71.3 \%)$ & $50(53.2 \%)$ & $42(44.7 \%)$ \\
High score & $60(63.8 \%)$ & $71(75.5 \%)$ & $27(28.7 \%)$ & $44(46.8 \%)$ & $52(55.3 \%)$ \\
\hline
\end{tabular}

alow score for stroma means moderate stroma, high score means dense stroma

95\% CI $1.084-3.553 ; P<0.001, \mathrm{HR}=4.027,95 \%$ CI 1.940-8.360). Finally, we conducted the multivariate analysis to investigate the prognostic factors together. Multivariate analysis showed that PD-1 TILs expression $(P=0.031 ; \mathrm{HR}=0.507 ; 95 \%$ CI $0.273-0.941)$, tumor differentiation $(P=0.006$; HR $=2.504 ; 95 \%$ CI $1.307-4.798)$ and vascular invasion $(P<0.001$; $\mathrm{HR}=5.931 ; 95 \%$ CI $2.657-13.238)$ were independent prognostic factors for OS of PC patients.

\section{Correlations of MMR gene expression, stroma density and PD-1/PD-L1 expression in PC tissues}

As shown in Table 5, an inverse correlation was identified between low expression of $\mathrm{MSH} 2$ and high expression of PD-L1 (Spearman correlation coefficient $\mathrm{r}=-0.295, P=0.004$, Table 5). No correlations were found between PD-L1 expression and MLH1 expression $(P>0.05$, Table 5) and between PD-L1 expression and stroma density $(P>0.05$, Table 5). Then, we used serial sections from the same PC tissue to depict the co-distribution of MSH2 and PD-L1 in PC tissue. As seen in Figure 3E, low MSH2 expression strongly correlated with high PD-L1 expression. For PD-1, as summarized in Table 6, we did not observe any correlation between PD-1 expression level and the expression of MMR gene ( $P>0.05$, Table 6). Also, PD-1 expression level was not associated with PD-L1 $(P>0.05$, Table 6). Then, we further made subgroup analyses to evaluate the prognostic impact of PD-1 expression stratified by PD-L1. Kaplan-Meier analysis and log-rank test result
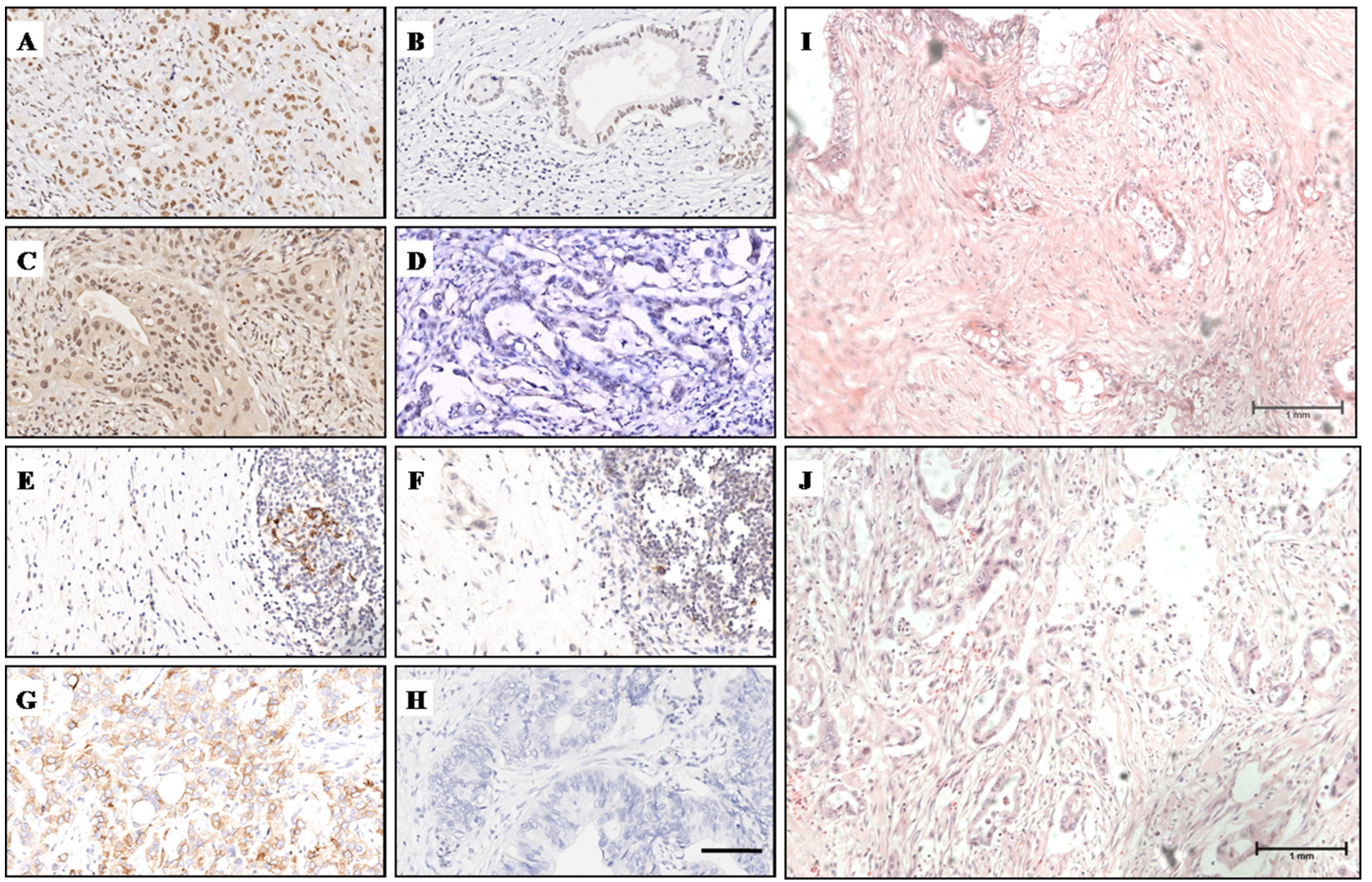

Figure 1: IHC and HE staining characteristics of MLH1, MSH2, PD-1, PD-L1 and stroma density. (A) High MLH1 expression was observed in the nucleus. (B) Low MLH1 expression. (C) High MSH2 expression was observed in the nucleus. (D) Low MSH2 expression. (E) PD-1 was located on cell menbranes of TILs, clusters of PD-1+ TILs were observed. (F) Sporadic PD-1+ TILs were observed. (G) High PD-1 expression was observed on cell menbranes of cancer cells. (H) Low PD-L1 expression. (I) H\&E staining showed PC tissue with dense stroma. (J) H\&E staining showed PC tissue with moderate stroma. Magnification, x200 (A-H); Magnification, x100 (I, J). 
Table 2: Correlation between the clinicopathologic characteristics and MLH1/MSH2 expression

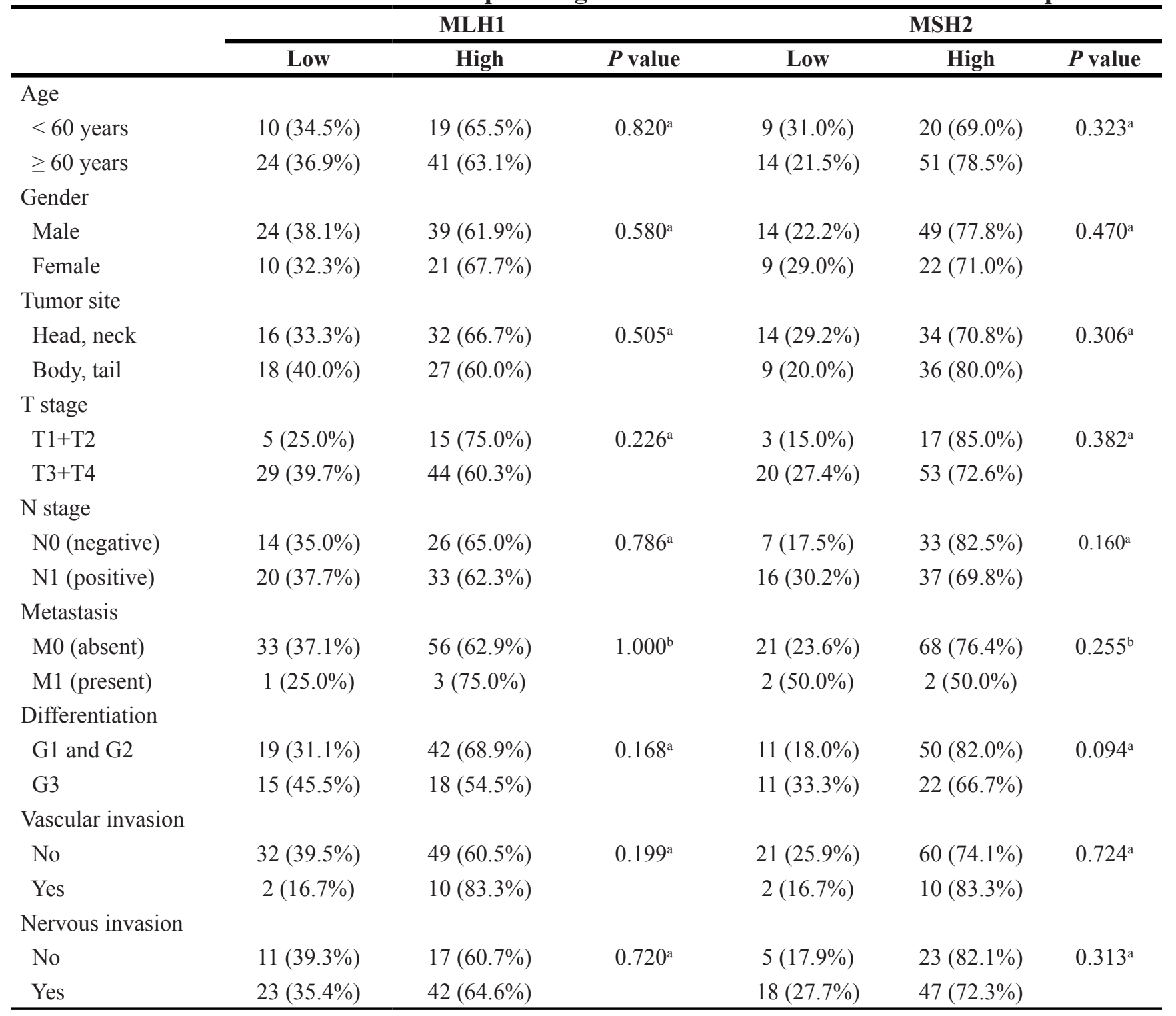

${ }^{\mathrm{a} C h i-s q u a r e}$ test; ${ }^{\mathrm{b}}$ Fisher's exact test

showed that PD-1 expression level was associated with OS only at low PD-L1 subgroup (Figure 3A, 2B, $P=0.021$ ). Moreover, high expression of PD-1 on cell membranes of TILs strongly correlated with moderate stroma $(P<0.001$, Table 6). Previous studies indicated that a better survival was seen in surgically resected PC patients with higher levels of TILs within the tumor microenvironment and dense stroma made PC tissue poor-vascularized and can prevent T-cell from infiltrating (Supplementary Figure S3). To further investigate the association of OS with PD-1 expression and stroma density, we stratified the cases into four subgroups based on PD-1 expression and stroma density (Figure 3C). Kaplan-Meier analysis showed that the OS of four subgroups had significant differences (log-rank test: $P=0.006$ ). Interesting, patients with high PD-1 expression and dense stroma had a better OS (median OS > 24 months), while patients with low PD-1 expression and moderate stroma showed a worst outcome (median OS $=7$ months). Likewise, when the first three subgroups were combined into a single subgroup named "all others", a similar result was found (log-rank test $P=0.006$, Figure 3D).

\section{DISCUSSION}

PD-1/PD-L1 axis, as an immune checkpoint, has been widely studied in many different types of malignant. The expression of PD-1/PD-L1 was often used as a biomarker of immune checkpoint inhibitors [21-22]. Moreover, PD-1/PD-L1 antibody has been shown to be highly effective in gastrointestinal cancers as a single agent in patients with mismatch repair deficiencies. Deficient MMR results in the incorporation of mismatched 

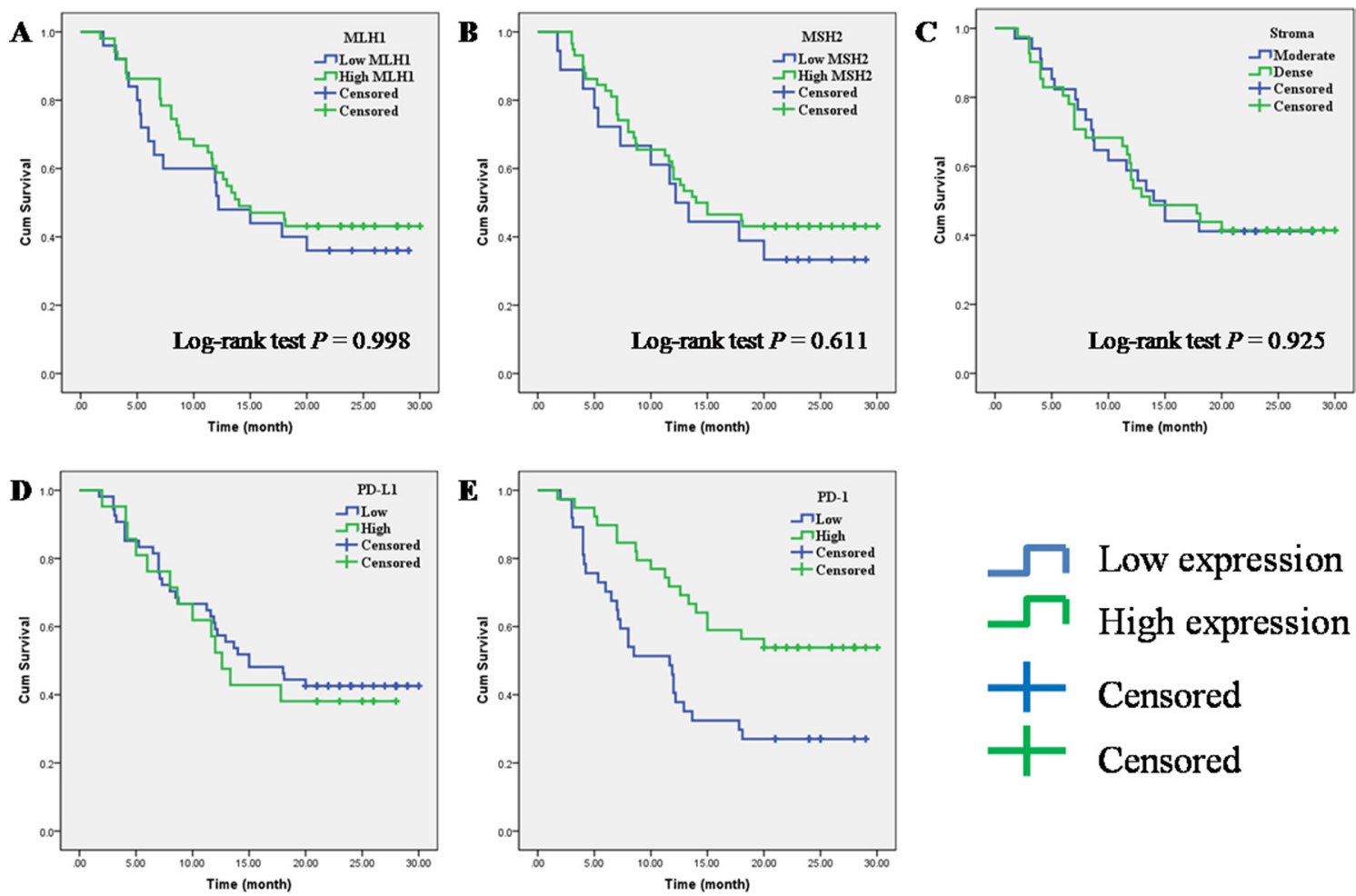
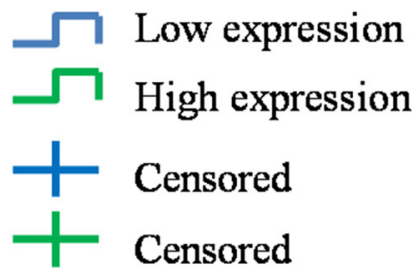

Figure 2: Prognostic impact of MLH1, MSH2, PD-1, PD-L1 and stroma density on overall survival in PC. (A) MLH1; (B) MSH2; (C) stroma density; (D) PD-L1; (E) PD-1.
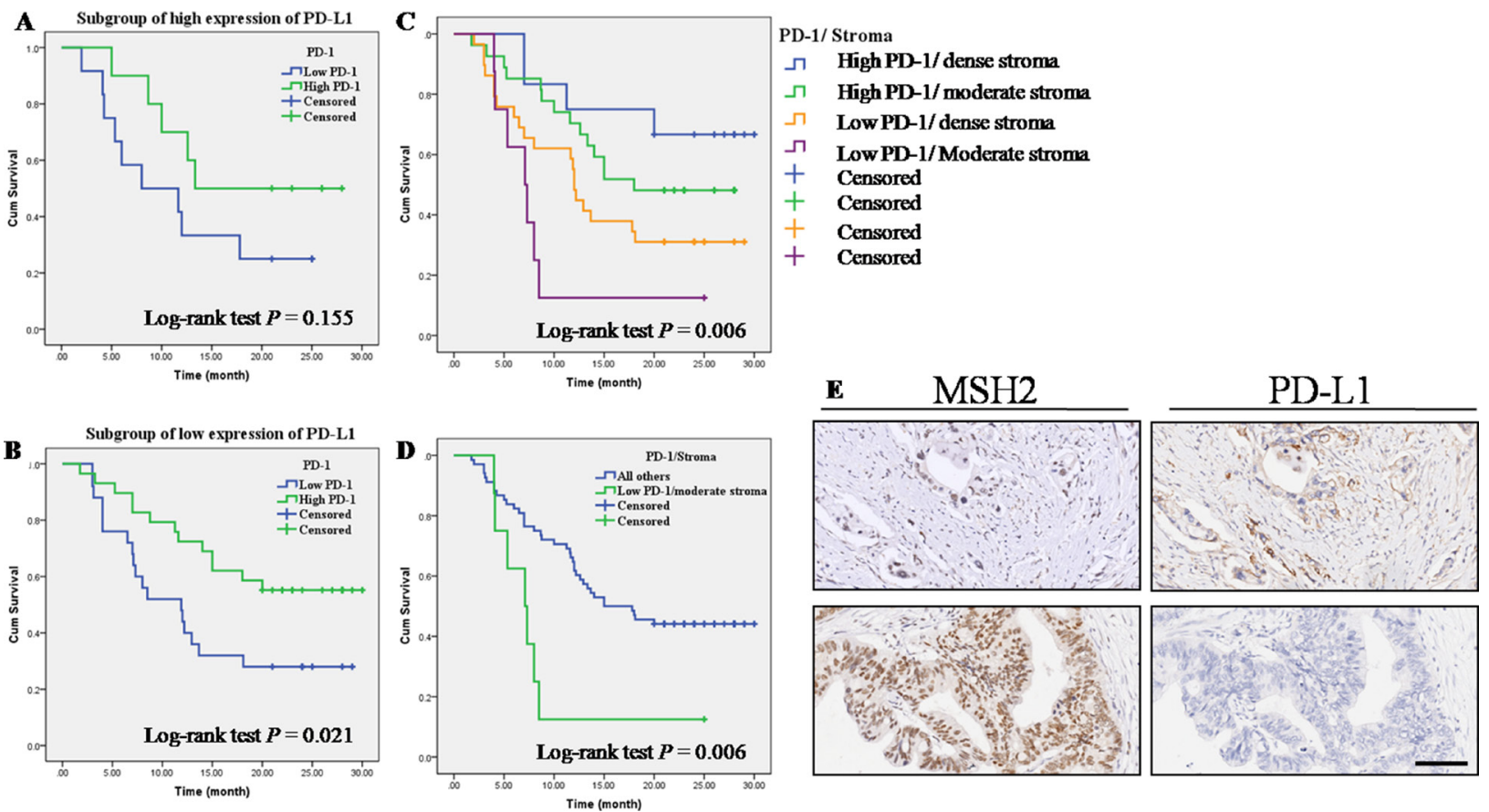

Figure 3: Correlations of MMR gene expression, stroma density and PD-1/PD-L1 expression in PC tissues. (A) OS according to PD-1 expression in high PD-L1 expression subgroup. (B) OS according to PD-1 expression in low PD-L1 expression subgroup. (C) OS in four subgroups straitified by PD-1 expression and stroma density. (D) Low PD-1 expression and moderate stroma showed a worse outcome than the combination of other three group named "all others". (E) Serial sections were used from the same PC tissue to depict an inverse correlation between MSH2 expression and PD-L1 expression. Top left and top right: low MSH2 expression vs. high PD-L1 expression; Lower left and lower right: high MSH2 expression vs. low PD-L1 expression. 
Table 3: Correlation between the clinicopathologic characteristics and PD-1/PD-L1 expression

\begin{tabular}{|c|c|c|c|c|c|c|c|c|c|}
\hline & \multicolumn{3}{|c|}{ PD-L1 } & \multicolumn{3}{|c|}{ PD-1 } & \multicolumn{3}{|c|}{ Stroma } \\
\hline & Low & High & $P$ value & Low & High & $P$ value & Moderate & Dense & $P$ value \\
\hline \multicolumn{10}{|l|}{ Age } \\
\hline$<60$ years & $19(65.5 \%)$ & $10(34.5 \%)$ & $0.410^{\mathrm{a}}$ & $16(55.2 \%)$ & $13(44.8 \%)$ & $0.797^{\mathrm{a}}$ & $13(44.8 \%)$ & $16(55.2)$ & $0.985^{\mathrm{a}}$ \\
\hline$\geq 60$ years & $48(73.8 \%)$ & $17(22.2 \%)$ & & $34(52.3 \%)$ & $31(47.7 \%)$ & & $29(44.6 \%)$ & $36(55.4 \%)$ & \\
\hline \multicolumn{10}{|l|}{ Gender } \\
\hline Male & $47(74.6 \%)$ & $16(25.4 \%)$ & $0.834^{\mathrm{a}}$ & $37(58.7 \%)$ & $26(41.3 \%)$ & $0.125^{\mathrm{a}}$ & $25(39.7 \%)$ & $38(60.3 \%)$ & $0.165^{\mathrm{a}}$ \\
\hline Female & $20(64.5 \%)$ & $11(35.5 \%)$ & & $13(41.9 \%)$ & $18(58.1 \%)$ & & $17(54.8 \%)$ & $14(45.2 \%)$ & \\
\hline \multicolumn{10}{|l|}{ Tumor site } \\
\hline Head, neck & $31(64.6 \%)$ & $17(35.4 \%)$ & $0.967^{\mathrm{a}}$ & $24(53.3 \%)$ & $21(46.7 \%)$ & $0.936^{\mathrm{a}}$ & $25(52.1 \%)$ & $23(47.9 \%)$ & $0.109^{\mathrm{a}}$ \\
\hline Body, tail & $35(77.8 \%)$ & $10(22.2 \%)$ & & $26(54.2 \%)$ & $22(45.8 \%)$ & & $16(35.6 \%)$ & $29(64.4 \%)$ & \\
\hline \multicolumn{10}{|l|}{ T stage } \\
\hline $\mathrm{T} 1+\mathrm{T} 2$ & $14(70.0 \%)$ & $6(30.0 \%)$ & $0.914^{\mathrm{a}}$ & $5(25.0 \%)$ & $15(75.0 \%)$ & $0.004^{a}$ & $12(60.0 \%)$ & $8(40.0 \%)$ & $0.106^{\mathrm{a}}$ \\
\hline $\mathrm{T} 3+\mathrm{T} 4$ & $52(71.2 \%)$ & $21(28.8 \%)$ & & $45(61.6 \%)$ & $28(38.4 \%)$ & & $29(39.7 \%)$ & $44(60.3 \%)$ & \\
\hline \multicolumn{10}{|l|}{$\mathrm{N}$ stage } \\
\hline N0 (negative) & $33(82.5 \%)$ & $7(17.5 \%)$ & $0.033^{\mathrm{a}}$ & $21(52.5 \%)$ & $19(47.5 \%)$ & $0.832^{\mathrm{a}}$ & $14(35.0 \%)$ & $26(65.0 \%)$ & $0.125^{\mathrm{a}}$ \\
\hline N1 (positive) & $33(62.3 \%)$ & $20(37.7 \%)$ & & $29(54.7 \%)$ & $24(45.3 \%)$ & & $27(50.9 \%)$ & $26(49.1 \%)$ & \\
\hline \multicolumn{10}{|l|}{ Metastasis } \\
\hline M0 (absent) & $63(70.8 \%)$ & $26(29.2 \%)$ & $0.999^{\mathrm{b}}$ & $50(56.2 \%)$ & $39(43.8 \%)$ & $0.324^{\mathrm{b}}$ & $38(42.7 \%)$ & $51(57.3 \%)$ & $0.317^{\mathrm{a}}$ \\
\hline M1 (present) & $3(75.0 \%)$ & $1(25.0 \%)$ & & $1(25.0 \%)$ & $3(75.0 \%)$ & & $3(75 \%)$ & $1(25 \%)$ & \\
\hline \multicolumn{10}{|l|}{ Differentiation } \\
\hline $\mathrm{G} 1$ and $\mathrm{G} 2$ & $49(80.3 \%)$ & $12(19.7 \%)$ & $0.008^{\mathrm{a}}$ & $27(44.3 \%)$ & $34(55.7 \%)$ & $0.018^{a}$ & $29(47.5 \%)$ & $32(52.5 \%)$ & $0.448^{\mathrm{a}}$ \\
\hline G3 & $18(54.5 \%)$ & $15(45.5 \%)$ & & $23(69.7 \%)$ & $10(30.3 \%)$ & & $13(39.4 \%)$ & $20(60.6 \%)$ & \\
\hline \multicolumn{10}{|c|}{ Vascular invasion } \\
\hline No & $56(69.1 \%)$ & $25(30.9 \%)$ & $0.498^{\mathrm{a}}$ & $43(53.1 \%)$ & $38(46.9 \%)$ & $0.734^{\mathrm{a}}$ & $33(40.7 \%)$ & $48(59.3)$ & $0.091^{\mathrm{a}}$ \\
\hline Yes & $10(83.3 \%)$ & $2(16.7 \%)$ & & $7(58.3 \%)$ & $5(41.7 \%)$ & & $8(66.7 \%)$ & $4(33.3 \%)$ & \\
\hline \multicolumn{10}{|c|}{ Nervous invasion } \\
\hline No & $23(82.1 \%)$ & $5(17.9 \%)$ & $0.119^{\mathrm{a}}$ & $14(50.0 \%)$ & $14(50.0 \%)$ & $0.633^{\mathrm{a}}$ & $12(42.9 \%)$ & $16(57.1 \%)$ & $0.876^{\mathrm{a}}$ \\
\hline Yes & $43(66.2 \%)$ & $22(33.8 \%)$ & & $36(55.4 \%)$ & $29(44.6 \%)$ & & $29(44.6 \%)$ & $36(55.4 \%)$ & \\
\hline
\end{tabular}

${ }^{\mathrm{a} C h i}$-square test; ${ }^{\mathrm{b}}$ Fisher's exact test;

Significant values $(P<0.05)$ have been marked with bold

nucleotides, with MMR-deficient colorectal cancer cells exhibiting 10-100 times the number of somatic mutations as those with proficient MMR [23-24]. Thus, high somatic mutation loads, which have the potential to create "non-self" immunogenic neoantigens, correlated with increased intratumoral effector $\mathrm{T}$ cell responses to the neoantigens Supplementary Tables S1, S2 [25]. Meanwhile, some studies indicated that MMR-deficient status was associated with higher expression of PD-L1 in gastric and colorectal cancer [26-28]. However, the relationship between MMR gene and PD-1/PD-L1 in pancreatic cancer remains largely unknown. Moreover, the correlations between PD-1/PD-L1 and prognosis are variants among different tumor types. Although Angela Diana et al. reported the expression of PD-1/PD-L1 and their prognostic value in pancreatic cancer [18]. However, the correlations of MMR-D/MMR-P and PD-1/PD-L1 and their clinical significant in pancreatic cancer were not fully understood, especially in East Asia.
Our study aimed at systematically analyzing the expression of MLH1, MSH2, PD-L1 and PD-1 in pancreatic cancer cells and tumor infiltrating cells, investigate their clinical significance and prognostic value. We found that high expression of PD-L1 on cancer cell membranes correlated with lymph node metastasis and strongly correlated with poor-differentiation, high expression of PD-1 on cell membranes of TILs correlated with well-differentiation and strongly correlated with advanced T stage, high PD-1 expression was associated with a significantly superior OS and was an independent prognostic factor. Then we found an inverse correlation between MSH2 expression and PD-L1 expression. In subgroup analyses, we observed that PD-1 expression level was associated with OS only at low PD-L1 expression subgroup. Finally, when we stratified the cases into four subgroups based on PD-1 expression and stroma density, we found that patients with high PD-1 expression and dense stroma had a better OS, while 
Table 4: Univariate and multivariate Cox regression analysis of overall survival

\begin{tabular}{|c|c|c|c|c|c|c|}
\hline & \multicolumn{3}{|c|}{ Univariate analysis } & \multicolumn{3}{|c|}{ Multivariate analysis } \\
\hline & HR & $95 \% \mathrm{CI}$ & $P$ value & HR & $95 \% \mathrm{CI}$ & $P$ value \\
\hline Low MLH1 & 1 & & & & & \\
\hline High MLH1 & 0.798 & $0.433-1.470$ & 0.470 & & & \\
\hline Low MSH2 & 1 & & & & & \\
\hline High MSH2 & 0.786 & $0406-1.523$ & 0.476 & & & \\
\hline Low PD-1 & 1 & & & & & \\
\hline High PD-1 & 0.441 & $0.242-0.804$ & 0.008 & 0.507 & $0.273-0.941$ & 0.031 \\
\hline Low PD-1L & 1 & & & & & \\
\hline High PD-1L & 1.204 & $0.640-2.264$ & 0.565 & & & \\
\hline Moderate Stroma & 1 & & & & & \\
\hline Dense Stroma & 0.972 & $0.541-1.747$ & 0.925 & & & \\
\hline Age $(<60$ years $)$ & 1 & & & & & \\
\hline Age ( $\geq 60$ years $)$ & 0.765 & $0.415-1.409$ & 0.390 & & & \\
\hline Gender (Male) & 1 & & & & & \\
\hline Gender (Female) & 0.896 & $0.482-1.666$ & 0.728 & & & \\
\hline Tumor site (Head, neck) & 1 & & & & & \\
\hline Tumor site (Body, tail) & 1.486 & $0.824-2.680$ & 0.188 & & & \\
\hline T stage (T1+T2) & 1 & & & & & \\
\hline T stage $(\mathrm{T} 3+\mathrm{T} 4)$ & 1.992 & $0.889-4.467$ & 0.094 & & & \\
\hline N stage (N0) & 1 & & & & & \\
\hline $\mathrm{N}$ stage (N1) & 1.212 & $0.670-2.191$ & 0.525 & & & \\
\hline Metastasis (M0) & 1 & & & & & \\
\hline Metastasis (M1) & 0.584 & $0.141-2.413$ & 0.457 & & & \\
\hline Differentiation (G1, G2) & 1 & & & & & \\
\hline Differentiation (G3) & 1.963 & $1.084-3.553$ & 0.026 & 2.504 & $1.307-4.798$ & 0.006 \\
\hline Vascular invasion (No) & 1 & & & & & \\
\hline Vascular invasion (Yes) & 4.027 & $1.940-8.360$ & $<0.001$ & 5.931 & $2.657-13.238$ & $<0.001$ \\
\hline Nervous invasion (No) & 1 & & & & & \\
\hline Nervous invasion (Yes) & 1.917 & $0.969-3.793$ & 0.062 & & & \\
\hline
\end{tabular}

HR: hazard ratio, $95 \%$ CI: 95\% confidence interval.

Significant values $(P<0.05)$ have been marked with bold.

patients with low PD-1 expression and moderate stroma showed a worst outcome.

In our study, high expression of PD-1 on cell membranes of TILs strongly correlated with advanced $\mathrm{T}$ stage. Our observations are in line with most of previous studies in pancreatic cancer [18]. Also, our study reported that high expression of PD-L1 correlated with lymph node metastasis and strongly correlated with poor-differentiation. In previous study, Guo Y et al. made a meta-analysis to evaluate the prognostic and clinicopathological value of PD-L1 in breast cancer [29]. The result showed that positive/higher PD-L1 expression was significantly associated with positive lymph node metastasis and poor nuclear grade.
Similar result was reported by Abbas $\mathrm{M}$ et al. in clear cell renal cell carcinoma [30]. The underline mechanisms remain unclear. Thus, part of the patients with metastatic lymph nodes, poorer nuclear grade may benefit from anti-PD-L1 therapy, which would improve their prognosis. PD-L1 expression was reported to be over expressed in MMR-deficient cancers, usually because MMR-deficient cancer creates high accumulated mutations which lead many tumor-specific neoantigens and is associated with high immune activities presumably aroused by mutated neoantigens. However, in present study, MSH2 was showed an inverse association with PD-L1 expression but not MLH1. These differences could be attributed to the several factors, such as the heterogeneity in population 
Table 5: Association between PD-L1 and other markers

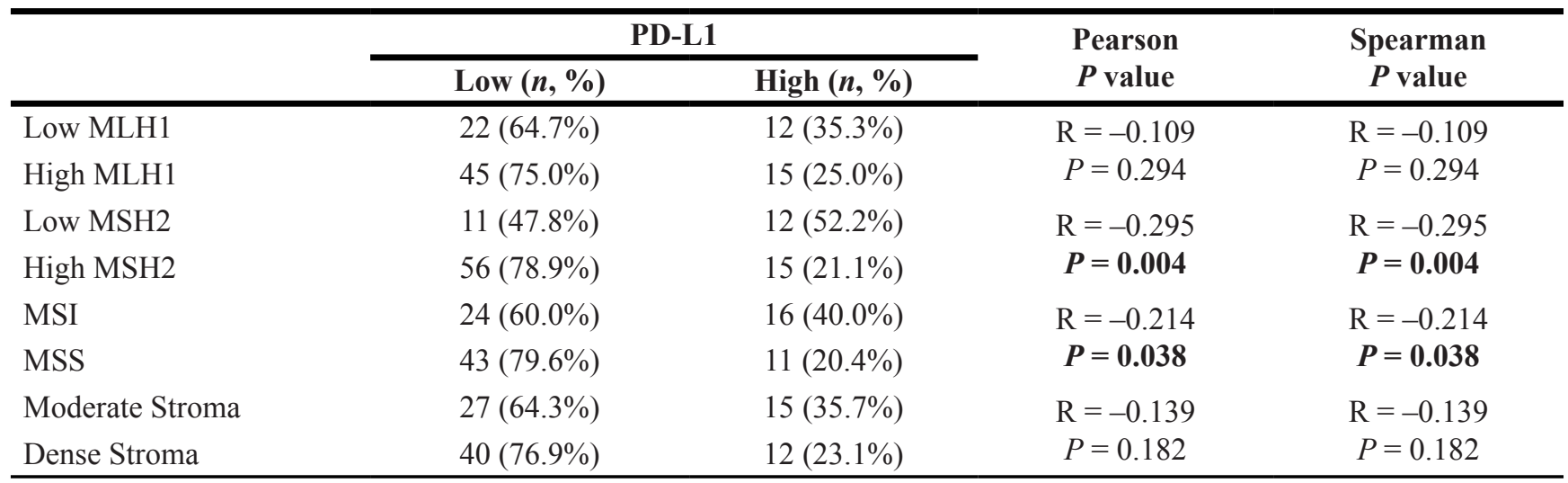

Significant values $(P<0.05)$ have been marked with bold.

Table 6: Association between PD-1 and other markers

\begin{tabular}{|c|c|c|c|c|}
\hline & \multicolumn{2}{|c|}{ PD-1 } & \multirow{2}{*}{$\begin{array}{c}\text { Pearson } \\
P \text { value }\end{array}$} & \multirow{2}{*}{$\begin{array}{c}\text { Spearman } \\
P \text { value }\end{array}$} \\
\hline & Low $(n, \%)$ & High $(n, \%)$ & & \\
\hline Low MLH1 & $21(61.8 \%)$ & $13(38.2 \%)$ & $\mathrm{R}=0.129$ & $\mathrm{R}=0.129$ \\
\hline High MLH1 & $29(48.3 \%)$ & $31(51.7 \%)$ & $P=0.214$ & $P=0.214$ \\
\hline Low MSH2 & $13(56.5 \%)$ & $10(43.5 \%)$ & $\mathrm{R}=0.038$ & $\mathrm{R}=0.038$ \\
\hline High MSH2 & $37(52.1 \%)$ & $34(47.9 \%)$ & $P=0.716$ & $P=0.716$ \\
\hline MMR-D & $23(57.5 \%)$ & $17(42.5 \%)$ & $\mathrm{R}=0.074$ & $\mathrm{R}=0.074$ \\
\hline MMR-P & $27(50.0 \%)$ & $27(50.0 \%)$ & $P=0.477$ & $P=0.477$ \\
\hline Moderate Stroma & $11(26.2 \%)$ & $31(73.8 \%)$ & $\mathrm{R}=-0.486$ & $\mathrm{R}=-0.486$ \\
\hline Dense Stroma & $39(75.0 \%)$ & $13(25.0 \%)$ & $P<0.001$ & $P<0.001$ \\
\hline Low PD-L1 & $34(50.7 \%)$ & $33(49.3 \%)$ & $\mathrm{R}=0.077$ & $\mathrm{R}=0.077$ \\
\hline High PD-L1 & $16(59.3 \%)$ & $11(40.7 \%)$ & $P=0.460$ & $P=0.460$ \\
\hline
\end{tabular}

Significant values $(P<0.05)$ have been marked with bold.

and different tumor type but also analysis method. In our study, high expression of PD-1 on cell membranes of TILs correlated with well-differentiation and a favorable clinical outcome in PC. Our results are in line with some studies in pancreatic cancer[18], but the mechanisms are currently unclear. Ashton et al. reported that antigenspecific immune activation following T-cell receptor stimulation leads to PD-1 upregulation on TILs [31]. Badoual et al. found higher expression of the immune activation markers HLA-DR and CD38 in PD-1+ TILs [32], so the presence of PD-1+ TILs could reflect an endogenous antitumor immune response that occurred upon activation of TILs. It could be an argument for this hypothesis that PD-1 expression level was associated with OS only at low PD-L1 expression subgroup. PC is characterised by an abundant desmoplastic stroma which is poor-vascularized and often prevents T-cell from infiltrating. The mechanisms include activated pancreatic stellate cells that drive apoptosis and sequestration of TILs, and the physical barrier imposed by dense collagen [33]. Analysis of PD-1+ TILs expression showed significantly higher infiltration in patients with moderate stroma density compared to patients with dense stroma and vice versa in our study. Moreover, patients with both low PD-1 expression and moderate stroma had a worse OS and vice versa. Intriguingly, Wang et al. reported stroma density constituted an independent prognostic marker in PC patients treated with adjuvant chemotherapy [8]. Some studies have reported higher TILs motility and migration in tumor areas with loose collagen [34]. Therefore, we speculated that low PD-1+ TILs in moderate stroma may reflect the lower TILs motility and migration and poor immune response of some patients. Obviously, these patients may have a worse outcome.

To our knowledge, the present study is the first study concerning PD-1/ PD-L1 and MMR statues analyses in East Asia population, which specifically investigated their clinical significant in PC. However, we would like to acknowledge the limitations of the study. Although we have analyzed a large cohort, the present study is a retrospective analysis and there is a potential for selection bias. And we did not include therapy 
Table 7: Detailed clinical information of patients in our study

\begin{tabular}{lcc}
\hline Overall survival & Categories & Numbers \\
Age & & 14 months, range (1.75-30.00) \\
Gender & Male & 62 years, range (31-78) \\
& Female & 63 \\
Tumor site & Head, neck & 31 \\
T stage & Body, tail & 48 \\
& T1+T2 & 45 \\
N stage & T3+T4 & 20 \\
& N0 (negative) & 73 \\
Metastasis & N1 (positive) & 40 \\
& M0 (absent) & 53 \\
Differentiation & M1 (present) & 89 \\
& G1 & 4 \\
Vascular invasion & G2 & 10 \\
& G3 & 51 \\
Nervous invasion & No & 33 \\
& Yes & 81 \\
\hline
\end{tabular}

responses in our studies which may affect the result of multivariate analysis.

In summary, we evaluated the correlation between clinicopathological characteristics and MMR, PD-1/PD-L1 expression, and found the prognosis value of PD-1 expression. In subgroup analyses, we observed that PD-1 expression level was associated with OS only at low PD-L1 expression subgroup. When we stratified the cases into four subgroups based on PD-1 expression and stroma density, we found that patients with high PD-1 expression and dense stroma had a better OS, while patients with low PD-1 expression and moderate stroma showed a worst outcome. Our result may provide more effective molecular markers for immunotherapeutic strategies of PC patients in clinical practice.

\section{MATERIALS AND METHODS}

\section{Patients and treatment}

This study was approved by the Ethics and Research Committees of Shanghai General Hospital, Shanghai Jiao Tong University School of Medicine, and was conducted in accordance with the Declaration of Helsinki Principles. The TMA used for this study includes 94 unselected, non-consecutive, primary, and sporadic PC treated between March 2012 and August 2014 in Pancreatic Cancer Center, Shanghai Jiao Tong University School of Medicine. Formalin-fixed, paraffin-embedded tissue blocks from resected PC were made. Tissue cylinders with a $2.0 \mathrm{~mm}$ diameter were punched from representative tissue areas. The histological types were confirmed by experienced pathologists. The TMAs contained well-documented clinicopathological information, including patients' age, sex, location, tumor differentiation, $T$ stage, lymph node metastasis, distant metastasis, nervous invasion, vascular invasion and follow-up data (ended in March, 2016). In total, 94 patients were included, 63 males and 31 females, with a median age of 62 years old (ranging from 31 to 78 years old). We got follow-up data of 77 patients in this cohort. The overall survival time ranged from 1.75 to 30.00 months, with a median of 14 months. Detailed information can be found in Table 7.

\section{Immunohistochemical staining}

Immunohistochemistry was performed based on the standard streptavidin-peroxidase (S-P) method (Zymed, San Francisco, CA). Briefly, the tissue microarrays were dewaxed and dehydrated in a xylene and alcohol bath solution. Heat mediated antigen retrieval was performed using Tris/EDTA buffer pH 9.0. After that, endogenous peroxidase activity was then blocked with $0.3 \%$ hydrogen peroxide for 10 mins. The slides were cooled to room temperature and blocked by incubating with normal goat serum at room temperature for $1 \mathrm{~h}$ and were subsequently incubated overnight at $4^{\circ} \mathrm{C}$ with primary antibodies as follows: PD-1 (dilution1:50, Abcam), PD-L1 (dilution 
1:500, Abcam), MLH1 (dilution1:500, Abcam), MSH2 (dilution 1:500, CST) (Supplementary Figure S2).

The sections were incubated with biotinylated secondary antibodies (Zymed, San Francisco, CA) for $30 \mathrm{~min}$ at room temperature, followed by incubation with streptavidin horseradish peroxidase complex. Finally, sections were visualized by 3, 3'-diaminobenzidine staining. Meanwhile, slides were stained with haematoxylin and eosin (H\&E) as described before.

\section{Scoring of immunohistochemistry}

Immunostaining signals were evaluated independently by two experienced pathologists without access to the patients' clinical and pathological features. MLH1, MSH2 and PD-L1 expression were scored according to staining intensity and the percentage of positive cells as previously described [35]. The staining intensity was scored as 0 (negative), 1 (weak), 2 (medium) or 3 (strong). The percentage of positive cells was scored as follows: $<5 \%(0), 5 \%-25 \%$ (1), $25 \%-50 \%$ (2), $50 \%-75 \%$ (3) and $75 \%-100 \%$ (4) according to the percentages of the positive staining areas in relation to the whole carcinoma area. Comprehensive score $=$ staining percentage $\times$ intensity.

Samples with a final staining score of $<6$ were classified as low expression, while those with score of $\geq 6$ were considered to be high expression. The extent of TILs was assessed in HE stained TMA. The expression of PD-1 in TILs by IHC method was evaluated by measuring cell density as previously reported. Scoring was as follows: (1) absent cells; (2) $<25 \%$ cell density; (3) $25-50 \%$ cell density; (4) $>50 \%$ cell density. Samples with a score of 1 or 2 were considered negative (low expression) and those with a score of 3 or 4 were considered positive (high expression). Stromal density based on HE staining, and classified as moderate or dense as previously described. The stromal density was evaluated on H\&E-stained TMAs. Quality was defined as moderate or strong on the basis of its morphologic appearance. Moderate stroma had a paucicellular matrix of loosely packed connective tissue fibres with occasional oedematous appearance. Cases with dense stroma showed a densely packed network of fibres with intense staining. Thus, dense stroma presents mature collagen fibres packed into multilayers with intense staining and others were considered to be "moderate" [7-8, 35].

\section{Statistical analysis}

All statistical analyses were carried out by the SPSS 16.0 software. The $\chi^{2}$ test and Fisher's exact test were used to analyze the correlations between categorical variables. Overall survival (OS) was defined as the interval from date of diagnosis until death from any cause. Data were censored for living patients and patients lost between follow-ups. The OS was estimated using the Kaplan-Meier method and compared using the log-rank test. Significant variables were further analyzed by multivariate analysis to test for independent prognosis. Bivariate correlations between variable factors were calculated by Spearman rank correlation coefficients. $P$-values $<0.05$ were considered statistically significant.

\section{ACKNOWLEDGMENTS AND FUNDING}

This study was supported in part by the Natural Science Foundation of China (grant NO. 81502017, $81502018,81572315,81101846,81171887$ and 91229117), by Research Grant from Shanghai Hospital Development Center (SHDC12014128), by National Key Clinical Discipline-Oncology, and by Songjiang Liandong Program(0702N14002).

\section{CONFLICTS OF INTEREST}

None.

\section{REFERENCES}

1. Torre LA, Bray F, Siegel RL, Ferlay J, Lortet-Tieulent J, Jemal A. Global cancer statistics, 2012. CA Cancer J Clin. 2015; 65:87-108.

2. Stathis A, Moore MJ. Advanced pancreatic carcinoma: current treatment and future challenges. Nat Rev Clin Oncol. 2010; 7:163-172.

3. Chen L. Co-inhibitory molecules of the B7-CD28 family in the control of T-cell immunity. Nat Rev Immunol. 2004; $4: 336-347$.

4. Topalian SL, Drake CG, Pardoll DM. Immune checkpoint blockade: a common denominator approach to cancer therapy. Cancer Cell. 2015; 27:450-461.

5. Topalian SL, Taube JM, Anders RA, Pardoll DM. Mechanismdriven biomarkers to guide immune checkpoint blockade in cancer therapy. Nat Rev Cancer. 2016; 16:275-287.

6. Neesse A, Algul H, Tuveson DA, Gress TM. Stromal biology and therapy in pancreatic cancer: a changing paradigm. Gut. 2015; 64:1476-1484.

7. Sinn M, Denkert C, Striefler JK, Pelzer U, Stieler JM, Bahra M, Lohneis P, Dorken B, Oettle H, Riess H, Sinn BV. alpha-Smooth muscle actin expression and desmoplastic stromal reaction in pancreatic cancer: results from the CONKO-001 study. Br J Cancer. 2014; 111:1917-1923.

8. Wang LM, Silva MA, D'Costa Z, Bockelmann R, Soonawalla Z, Liu S, O'Neill E, Mukherjee S, McKenna WG, Muschel R, Fokas E. The prognostic role of desmoplastic stroma in pancreatic ductal adenocarcinoma. Oncotarget. 2016; 7:4183-4194. doi: 10.18632/oncotarget.6770.

9. Fokas E, O'Neill E, Gordon-Weeks A, Mukherjee S, McKenna WG, Muschel RJ. Pancreatic ductal adenocarcinoma: From genetics to biology to radiobiology 
to oncoimmunology and all the way back to the clinic. Biochim Biophys Acta. 2015; 1855:61-82.

10. Brahmer JR, Tykodi SS, Chow LQ, Hwu WJ, Topalian SL, Hwu P, Drake CG, Camacho LH, Kauh J, Odunsi K, Pitot HC, Hamid O, Bhatia S, et al. Safety and activity of anti-PD-L1 antibody in patients with advanced cancer. N Engl J Med. 2012; 366:2455-2465.

11. Rizvi NA, Mazieres J, Planchard D, Stinchcombe TE, Dy GK, Antonia SJ, Horn L, Lena H, Minenza E, Mennecier B, Otterson GA, Campos LT, Gandara DR, et al. Activity and safety of nivolumab, an anti-PD-1 immune checkpoint inhibitor, for patients with advanced, refractory squamous non-small-cell lung cancer (CheckMate 063): a phase 2, single-arm trial. Lancet Oncol. 2015; 16:257-265.

12. Topalian SL, Hodi FS, Brahmer JR, Gettinger SN, Smith DC, McDermott DF, Powderly JD, Carvajal RD, Sosman JA, Atkins MB, Leming PD, Spigel DR, Antonia SJ, et al. Safety, activity, and immune correlates of anti-PD-1 antibody in cancer. N Engl J Med. 2012; 366:2443-2454.

13. Wolchok JD, Kluger H, Callahan MK, Postow MA, Rizvi NA, Lesokhin AM, Segal NH, Ariyan CE, Gordon RA, Reed K, Burke MM, Caldwell A, Kronenberg SA, et al. Nivolumab plus ipilimumab in advanced melanoma. N Engl J Med. 2013; 369:122-133.

14. NH Segal SJA, JR Brahmer, M Maio, A Blake-Haskins, et al. Preliminary data from a multi-arm expansion study of MEDI4736, an anti-PD-L1 antibody. J Clin Oncol. 2014; 32:5s (abstract 3002).

15. Lee V, Murphy A, Le DT, Diaz LA, Jr. Mismatch Repair Deficiency and Response to Immune Checkpoint Blockade. Oncologist. 2016.

16. Diaz LA, Jr. and Le DT. PD-1 Blockade in Tumors with Mismatch-Repair Deficiency. N Engl J Med. 2015; 373:1979.

17. Sijmons RH, Hofstra RM. Review: Clinical aspects of hereditary DNA Mismatch repair gene mutations. DNA Repair (Amst). 2016; 38:155-162.

18. Diana A, Wang LM, D'Costa Z, Allen P, Azad A, Silva MA, Soonawalla Z, Liu S, McKenna WG, Muschel RJ, Fokas E. Prognostic value, localization and correlation of PD-1/ PD-L1, CD8 and FOXP3 with the desmoplastic stroma in pancreatic ductal adenocarcinoma. Oncotarget. 2016; 7:40992-41004. doi: 10.18632/oncotarget.10038.

19. Li Y, Liang L, Dai W, Cai G, Xu Y, Li X, Li Q, Cai S. Prognostic impact of programed cell death-1 (PD-1) and PD-ligand 1 (PD-L1) expression in cancer cells and tumor infiltrating lymphocytes in colorectal cancer. Mol Cancer. 2016; 15:55.

20. Boland JM, Kwon ED, Harrington SM, Wampfler JA, Tang H, Yang P, Aubry MC. Tumor B7-H1 and B7-H3 expression in squamous cell carcinoma of the lung. Clin Lung Cancer. 2013; 14:157-163.

21. Taube JM, Klein A, Brahmer JR, Xu H, Pan X, Kim JH, Chen L, Pardoll DM, Topalian SL, Anders RA. Association of PD-1, PD-1 ligands, and other features of the tumor immune microenvironment with response to anti-PD-1 therapy. Clin Cancer Res. 2014; 20:5064-5074.

22. Powles T, Eder JP, Fine GD, Braiteh FS, Loriot Y, Cruz C, Bellmunt J, Burris HA, Petrylak DP, Teng SL, Shen X, Boyd Z, Hegde PS, et al. MPDL3280A (anti-PD-L1) treatment leads to clinical activity in metastatic bladder cancer. Nature. 2014; 515:558-562.

23. Timmermann B, Kerick M, Roehr C, Fischer A, Isau M, Boerno ST, Wunderlich A, Barmeyer C, Seemann P, Koenig J, Lappe M, Kuss AW, Garshasbi M, et al. Somatic mutation profiles of MSI, MSS colorectal cancer identified by whole exome next generation sequencing and bioinformatics analysis. PLoS One. 2010; 5:e15661.

24. Jin Z, Yoon HH. The promise of PD-1 inhibitors in gastroesophageal cancers: microsatellite instability vs. PD-L1. J Gastrointest Oncol. 2016; 7:771-788.

25. Bupathi $\mathrm{M}, \mathrm{Wu} \mathrm{C}$. Biomarkers for immune therapy in colorectal cancer: mismatch-repair deficiency and others. J Gastrointest Oncol. 2016; 7:713-720.

26. Inaguma S, Lasota J, Wang Z, Felisiak-Golabek A, Ikeda H, Miettinen M. Clinicopathologic profile, immunophenotype, and genotype of CD274 (PD-L1)-positive colorectal carcinomas. Mod Pathol. 2016.

27. Toh JW, de Souza P, Lim SH, Singh P, Chua W, Ng W, Spring KJ. The Potential Value of Immunotherapy in Colorectal Cancers: Review of the Evidence for Programmed Death-1 Inhibitor Therapy. Clin Colorectal Cancer. 2016.

28. Kawazoe A, Kuwata T, Kuboki Y, Shitara K, Nagatsuma AK, Aizawa M, Yoshino T, Doi T, Ohtsu A, Ochiai A. Clinicopathological features of programmed death ligand 1 expression with tumor-infiltrating lymphocyte, mismatch repair, and Epstein-Barr virus status in a large cohort of gastric cancer patients. Gastric Cancer. 2016.

29. Guo Y, Yu P, Liu Z, Maimaiti Y, Wang S, Yin X, Liu C, Huang T. Prognostic and Clinicopathological Value of Programmed Death Ligand-1 in Breast Cancer: A MetaAnalysis. PLoS One. 2016; 11:e0156323.

30. Abbas M, Steffens S, Bellut M, Eggers H, Grosshennig A, Becker JU, Wegener G, Schrader AJ, Grunwald V, Ivanyi P. Intratumoral expression of programmed death ligand 1 (PD-L1) in patients with clear cell renal cell carcinoma (ccRCC). Med Oncol. 2016; 33:80.

31. Ashton N, Evans RL, Elliott AC, Green R, Argent BE. Regulation of fluid secretion and intracellular messengers in isolated rat pancreatic ducts by acetylcholine. J Physiol. 1993; 471:549-562.

32. Badoual C, Hans S, Merillon N, Van Ryswick C, Ravel P, Benhamouda N, Levionnois E, Nizard M, Si-Mohamed A, Besnier N, Gey A, Rotem-Yehudar R, Pere H, et al. PD1-expressing tumor-infiltrating $\mathrm{T}$ cells are a favorable prognostic biomarker in HPV-associated head and neck cancer. Cancer Res. 2013; 73:128-138. 
33. Ene-Obong A, Clear AJ, Watt J, Wang J, Fatah R, Riches JC, Marshall JF, Chin-Aleong J, Chelala C, Gribben JG, Ramsay AG, Kocher HM. Activated pancreatic stellate cells sequester $\mathrm{CD} 8+\mathrm{T}$ cells to reduce their infiltration of the juxtatumoral compartment of pancreatic ductal adenocarcinoma. Gastroenterology. 2013; 145:1121-1132.

34. Hartmann N, Giese NA, Giese T, Poschke I, Offringa R, Werner J, Ryschich E. Prevailing role of contact guidance in intrastromal T-cell trapping in human pancreatic cancer. Clin Cancer Res. 2014; 20:3422-3433.

35. Ueno H, Jones AM, Wilkinson KH, Jass JR, Talbot IC. Histological categorisation of fibrotic cancer stroma in advanced rectal cancer. Gut. 2004; 53:581-586. 